\section{The free classification of analyzable and unanalyzable stimuli*}

\author{
STEPHEN HANDEL $\dagger$ \\ University of Tennessee, Knoxville, Tennessee 37916 \\ and \\ SHIRO IMAI \\ University of Hokkaido, Sapporo, Japan
}

The free classification of two-dimensional analyzable and unanalyzable stimuli was investigated. Analyzable stimuli consist of dimensions which are distinct and phenomenally separable (e.g., colored geometric forms); unanalyzable stimuli consist of two dimensions which are not distinct, not phenomenally separable, and which are probably perceived as a unitary dimension (e.g., Munsell colors). In the first experiment, Ss judged the similarity between each stimulus pair for both types of stimuli. The results replicated previous work in showing that the city-block metric was appropriate for the analyzable stimuli and that the euclidean metric was appropriate for unanalyzable stimuli. In the second experiment, Ss classified sets of analyzable and unanalyzable stimuli into two or three groups in any way they wished. For analyzable stimuli, classification was based on the dimensional structure-stimuli which alter the dimensional structure by defining another dimension change classification. For unanalyzable stimuli, classification was based on the similarity structure-stimuli which alter the similarity structure change classification.

One enduring issue in perception has been to determine how observers perceive multidimensional stimuli. It appears that no single answer is possible, largely because the perception of a multidimensional stimulus seems to depend on the nature of its dimensions. Stimuli may be composed of analyzable dimensions where each dimension is obvious and where all dimensions are easily pulled apart. Stimuli may be composed of unanalyzable dimensions where each dimension is less distinct and where all dimensions are seen as forming a unitary whole. Unanalyzable stimuli which, while constructed out of more than a single dimension, would seem to be perceived as differing on only one admittedly complex dimension. It must be emphasized that a dimension may seem to be analyzable when paired with one dimension but unanalyzable when paired with a different second dimension. For this reason, it makes more sense to talk about analyzable and unanalyzable stimuli rather than analyzable and unanalyzable dimensions.

Work using similarity judgments has shown that the distance metric of a set of multidimensional stimuli varies with the nature of the dimensions. For analyzable stimuli, the city-block metric seems appropriate, while for unanalyzable stimuli the euclidean metric seems appropriate (Torgerson, 1958; Hyman \& Well, 1967, 1968). These results lead to the tentative

* This research was supported in part by NIH Grant MH 15969.

†Address: Department of Psychology, Ayres Hall, University of Tennessee, Knoxville, Tennessee 37916 . conclusion that for analyzable stimuli, Ss attend selectively to dimensions, summing the perceived distances along each dimension, while for unanalyzable stimuli, Ss seem to make judgments of perceived distance directly.

In the present experiment, several types of two-dimensional stimuli thought to be representative of analyzable and unanalyzable stimuli were constructed. In Experiment 1, Ss judged the similarity between all pairs of stimuli for each type of two-dimensional stimulus. These judgments were used to calculate the best-fitting distance metric so as to see if stimuli thought to be analyzable were best represented by the city-block metric and stimuli thought to be unanalyzable were best represented by euclidean metric. If so, then these stimuli may be used to investigate how analyzability affects other perceptual tasks such as stimulus classification. In Experiment 2, Ss actually classified sets of three or four stimuli for each type of stimulus to determine the effect of stimulus analyzability on classification.

\section{STIMULI}

The stimuli used are shown schematically in Fig. 1. The levels of each dimension are represented by the rows or columns of the 4 by 4 matrix.

The dimensions of the first type of stimulus were shape and color (identical to those dimensions used by Imai \& Garner, 1968). These stimuli were thought to be analyzable, the levels of each dimension were nominal, and the discriminability (i.e., dissimilarity among levels) was high. The stimuli were made from colored construction paper. All forms were of equal subjective size (average area was $4.5 \mathrm{~cm}^{2}$ ) and were mounted on white matte cardboard backing measuring $5 \times 9 \mathrm{~cm}$.

The dimensions of the second and third type of stimulus were size of square and achromatic Munsell lightness (varying from white to black). We will use the term lightness instead of the proper Munsell value term to avoid confusion with the fourth type of stimulus. These stimuli were thought to be analyzable, the levels of each dimension could be placed on an interval scale (i.e., scalable), and discriminability was high for the second dimension pair and low for the third dimension pair. The squares were cut from glossy Munsell paper. The stimuli were not mounted, since a background might have affected their perception.

The dimensions of the fourth stimulus type were Munsell value and chroma; all chips were the same hue-5RP. In the present paper, the stimuli will be labeled by their /value/chroma notation. These dimensions were thought to be unanalyzable, the levels of each dimension could be scaled, and the distance between levels on each dimension was maximal. The chips were glossy $(2.5 \times 3.7 \mathrm{~cm})$ and mounted on white matte cardboard backing ( $5 \times 9 \mathrm{~cm}$ ).

The magnitudes of the levels of the dimensions for the size by lightness and value by chroma stimuli were chosen in a preliminary experiment to equate differences between levels of each dimension pair. This procedure was not used for the shape by color stimuli, because Imai and Garner (1968) had found that differences between simple geometric forms were about equal to differences between primary colors.

\section{EXPERIMENT 1:}

\section{SIMILARITY JUDGMENTS}

The aim of this experiment was: (1) to confirm that the shape by color and the size by lightness stimuli were analyzable by showing that the city-block metric best represented the similarity judgments and that the value by chroma stimuli were unanalyzable by showing that the euclidean metric best represented the similarity judgments; and (2) to obtain similarity judgments for use in investigating classifications in Experiment 2.

\section{Method 1}

Subjects. The Ss were 16 Kansas State University undergraduates with normal color vision.

Procedure. For each dimension pair 

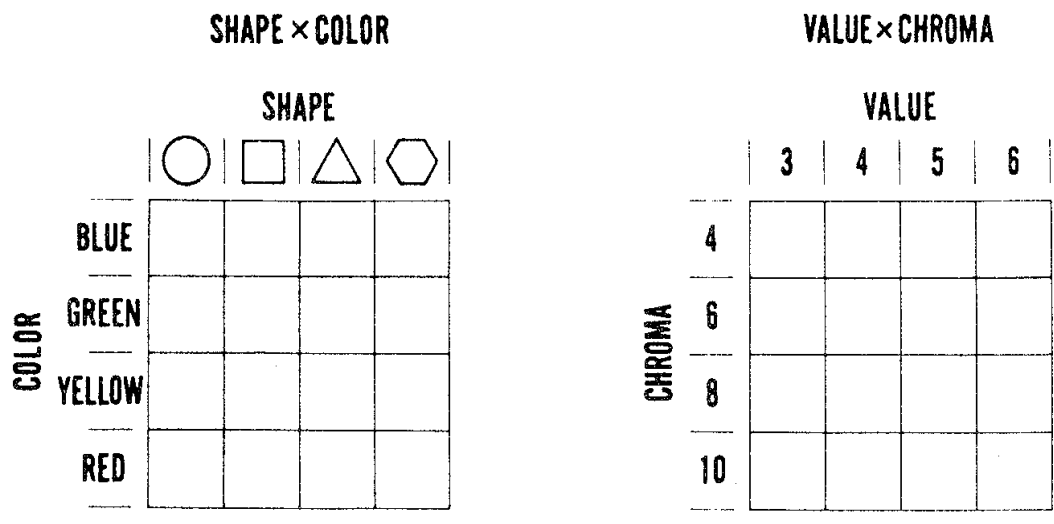

\section{SIZE $\times$ LIGHTNESS}

\section{HIGH DISCRIMINABILITY}

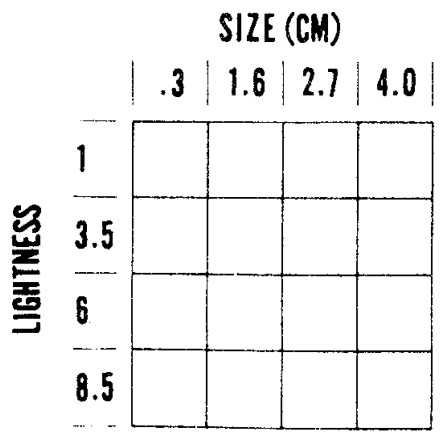

\section{SIZE $\times$ LIGHTNESS}
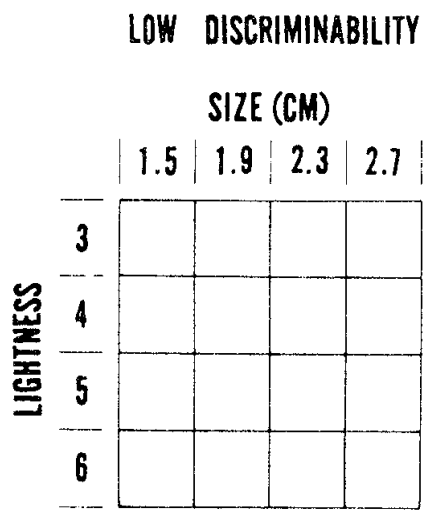

Fig. 1. A two-dimensional matrix diagram of each dimension pair. The levels of each dimension are represented by the rows or columns of the matrix.

there were 120 possible pairs of stimuli. Using Fig. 1 as a schematic, each stimulus pair can be represented by placing dots in 2 of the 16 cells of the 4 by 4 matrix. Thus, two dots in the first two rows of the first column would represent the stimulus pair blue circle/green circle for the shape by color stimuli or the stimulus pair $/ 3 / 4-13 / 6$ for the value by chroma stimuli.

Each dimension pair was the result of the factorial combination of two four-level dimensions. If the dimensions are independent and if the differences between levels of dimensions are equated, then many stimulus pairs are logically identical (i.e., measuring the same stimulus difference). If the dimensions are independent, then the difference between two levels of one dimension should not vary as a function of the second dimension. For example, the difference between Chroma 4 and Chroma 6 should be identical for Values $3,4,5$, and 6 . If differences between levels of each dimension are equated, then the similarity between two stimuli should depend only on the type of difference. As an example of a one-dimensional difference, the similarity between Value 3 and Value 5 should equal the similarity between Value 4 and Value 6 for all chromas (eight stimulus pairs measure differences of two levels of the value dimension). As an example of a two-dimensional difference, the similarity between stimuli $/ 3 / 4$ and /4/8 should equal that between /5/6 and $/ 6 / 10$ (12 stimulus pairs measure differences of one level of value and two levels of chroma). Finally, if the differences between levels on each dimension were equal, then spacing should be the sole determinant of similarity and the magnitudes of the differences on each dimension may be interchanged. That is, the difference between $/ 3 / 4$ and $/ 4 / 8$ should logically be equal to that between $/ 4 / 6$ and $16 / 4$.

On the assumption that the dimensions were independent and that the differences among levels were equated (evidence for this point will be presented in the results section), the 120 pairs were organized into 15 logically different pairs. The 15 pairs are shown in Fig. 2, with the number of instances shown in parenthesis. Two logical pairs which are equivalent if the differences among levels of the two dimensions were equated are shown together, one pair represented by dots and the other by xs.

Each $S$ judged the similarity between 60 pairs of stimuli for one type of stimulus. One-half of the instances for each logical pair was presented to each $S$. The $S$ s rated similarity on a 10-point scale, with "1" representing a very similar pair and " 10 " representing a very different pair.

Four Ss judged each dimension pair so that each instance of a logical pair was rated by two different Ss. The 60 judged pairs differed for each $S$, and their order of presentation was counterbalanced. In order to stabilize judgments, Ss judged 10 nonexperimental pairs before the 60 experimental pairs. These pairs were picked to display the range of similarity.

The E kept the 16 stimuli of each dimension pair behind a partition and placed 2 stimuli in front of $S$. The $S$ verbally stated his judgment, and then the next pair was presented. The task was self-paced.

\section{Results}

The average similarity and standard deviation of the judgments for each logical stimulus pair are shown in Table 1 for the shape by color stimuli and in Fig. 2 for the size by lightness and the value by chroma stimuli.

For all types of two-dimensional stimuli, there was no difference in judged similarity among the instances of each logical pair; judged similarity was a function only of the type of difference between stimuli. The similarity judgments were therefore averaged across instances.

For the shape by color stimuli, further analyses using uncorrelated $t$ tests indicated that all four colors were equally similar (2.4), all four geometric shapes were equally similar (4.8), and the similarity between all pairs of stimuli differing on both dimensions was also equal (6.5). Stimuli differing on two dimensions were always judged more dissimilar than stimulj differing on one dimension.

For the size by lightness and the value by chroma stimuli, further analyses investigated whether differences among levels of each dimension were equated. If so, similarity should depend solely on the spacing between stimuli for both oneand two-dimensional differences. There are six corresponding stimulus 


\begin{tabular}{|c|c|c|c|c|c|c|c|}
\hline \multicolumn{2}{|c|}{ LOGICAL PAIR } & \multicolumn{6}{|c|}{ SIMILARITY JUDGMENT } \\
\hline STIMULUS & TYPE & \multicolumn{4}{|c|}{$\begin{array}{l}\text { SIZE } \times \text { LIGHTNESS } \\
\text { DISCRIMINABILITY }\end{array}$} & \multicolumn{2}{|c|}{$\begin{array}{l}\text { VALUE } \\
\times\end{array}$} \\
\hline & or & HI & & $\mathrm{L}$ & & & \\
\hline & DITRESLENG & $\bar{x}$ & S.D. & $\bar{x}$ & S.D. & $\bar{x}$ & S.D. \\
\hline $\begin{array}{c}1,2 \\
(12,12)\end{array}$ & & 2.8 & .9 & 2.3 & .4 & 1.8 & .6 \\
\hline $\begin{array}{c}3,4 \\
(8,8)\end{array}$ & & 3.5 & 1.5 & 3.7 & 1.3 & 3.9 & 1.7 \\
\hline $\begin{array}{c}5,6 \\
(4,4)\end{array}$ & & 5.5 & 2.7 & 4.4 & 1.1 & 5.1 & 1.4 \\
\hline $\begin{array}{c}7 \\
(18)\end{array}$ & & 4.1 & 1.1 & 3.4 & .8 & 2.6 & .9 \\
\hline $\begin{array}{c}8 \\
(8)\end{array}$ & & 6.2 & 1.4 & 6.4 & 1.3 & 6.0 & 2.4 \\
\hline $\begin{array}{c}9 \\
(2)\end{array}$ & & 10.0 & .0 & 9.3 & 1.0 & 8.5 & 1.0 \\
\hline $\begin{array}{l}10,11 \\
(12,12)\end{array}$ & ex & 5.2 & 1.2 & 4.8 & 1.4 & 4.4 & 1.3 \\
\hline $\begin{array}{l}12,13 \\
(6,6)\end{array}$ & & 6.6 & 1.6 & 6.3 & 1.1 & 7.3 & 1.9 \\
\hline $\begin{array}{l}14,15 \\
(4,4)\end{array}$ & {$[x$} & 8.3 & 1.4 & 8.3 & 1.4 & 7.1 & 1.7 \\
\hline
\end{tabular}

Fig. 2. The mean similarity judgments for each pair of stimuli with " 1 " being highly similar and "10" being highly dissimilar. The logically different pairs are numbered in the first column with the number of instances below. The logical pairs are illustrated in the second column. For logical pairs which are equivalent if dimensions are equated, one pair is denoted by two dots and the other by two xs.

pairs if dimensions were equated. Of 18 comparisons ( 6 pairs by 3 dimension pairs), in no instance did similarity differ significantly (uncorrelated $t$ tests). Similarity judgments were therefore averaged across corresponding pairs.

\section{Discussion}

The obtained similarity judgments confirmed the expectations that the value, chroma, size, and lightness dimensions were scalable. Similarity was monotonic to physical distance, so that increasing the physical distance between a pair of stimuli decreased judged similarity. Furthermore, the discriminability of pairs of dimensions was equal. The shape and color dimensions possess only nominal properties; all shape differences and all color differences were equal, and all two-dimensional differences were equal and greater than one-dimensional differences.

Euclidean or city-block metric? To determine which distance metric was most appropriate, it was assumed that the similarity judgments of one-dimensional differences were accurate, and these were used to predict the two-dimensional differences. Distance exponents for Minkowski metrics ranging from 1 to 2-the exponent for the city-block metric being 1 and the exponent for the euclidean metric being 2-were used to predict the two-dimensional differences. The measure of fit was the sum of the absolute deviations between the actual and predicted two-dimensional similarities.

For the shape by color stimuli, the distance exponent which best predicted the judgments was 1.2 ; for the high discriminability size by lightness stimuli, the exponent was 1.2; for the low discriminability size by lightness stimuli, the exponent was 1.1 ; and for the value by chroma stimuli, the exponent was 1.7. For all stimuli, the best distance metric was between the euclidean and city block. The metric for the value by chroma stimuli was closer to the euclidean metric, while the metric for all other dimension pairs closely resembled the city-block metric [see Micko \& Fisher (1970) for a plausible explanation of metrics between 1 and 2 ].

A different approach also confirms these conclusions. Assume that the levels of dimensions are equally spaced. Then the expected similarity of each logical pair for either metric can be ranked. If the city-block metric is appropriate, the ranking from highest to lowest similarity would be $(1,2),[(3,4), 7], \quad[(5,6),(10,11)]$, $[8,(12,13)],(14,15), 9$, with the predicted similarity of pairs within brackets being equal. If the euclidean metric is appropriate, the ranking would be $(1,2), 7,(3,4),(10,11)$, $(5,6), 8,(12,13),(14,15), 9$. For the city-block metric, there are three cases in which two different logical pairs would be judged equally similar. This is due to the additivity of distances for the city-block metric [e.g., a difference of two levels on one dimension is equal to a difference of one level on two dimensions, Type $(3,4)$ equals Type 7]. But for the euclidean metric, there is a prediction as to which logical pair should be more similar for all three cases.

For the value by chroma stimuli, all three cases order as the euclidean metric would predict; for the high

Table 1

Similarity Judgments for the Shape by Color Dimensions

\begin{tabular}{lcccr}
\multicolumn{1}{c}{ Differences } & $\begin{array}{c}\text { Logical } \\
\text { Pairs }\end{array}$ & $\begin{array}{c}\text { Number of } \\
\text { Instances }\end{array}$ & Mean & SD \\
\hline Shape & $1,3,5$ & 24 & 4.8 & 2.0 \\
Color & $2,4,6$ & 24 & 2.4 & 1.2 \\
Shape and color & $7-15$ & 72 & 6.5 & 1.8 \\
\hline
\end{tabular}


discriminability size by lightness stimuli, two cases order as the euclidean metric would predict; and for the low discriminability size by lightness stimuli, only one case orders as the euclidean metric would predict. In two cases, the euclidean metric would predict that two-dimensional differences will be more similar than one-dimensional differences. But for the shape by color stimuli, all one-dimensional differences were more similar than two-dimensional differences.

\section{Conclusions}

The shape by color and the size by lightness stimuli appear to be analyzable: the dimensions are phenomenally separable, and the similarity judgments were best represented by the city-block metric. Discriminability did not affect the analyzability of the size by lightness stimuli. The value by chroma stimulj appear to be unanalyzable: the dimensions are not phenomenally separable, and the similarity judgments were best represented by the euclidean metric.

\section{EXPERIMENT 2:}

\section{FREE CLASSIFICATION}

In a classification task, $S$ sorts a set of stimuli into discrete groups in any way he wishes. The $S$ can use any number of groups (free classification), or $\mathrm{S}$ must use a specified number of groups (restricted classification). For both free and restricted classification, $S$ is free to place each stimulus in any group.

In performing a task of this type, $S$ may classify on the basis of the dimensional or similarity structure. For dimensional classification, one group would be formed for each level of one dimension so that stimuli within each group would necessarily differ on the other dimensions. For similarity classification, similar stimuli would be placed in the same group and dissimilar stimuli placed in different groups. The classification would maximize between-group dissimilarity and maximize within-group similarity. Furthermore, if sets can be dimensionally classified using either dimension, Ss may simply prefer to use one dimension for classification regardless of similarity, or Ss may choose the dimension used for classification when the levels of that dimension are most dissimilar (i.e., classification by shape if shape differences are greater than color differences)

Previous research has suggested that geometric stimuli are classified by dimensions, although Munsell colors are classified by similarity. For geometric shapes, the classification of nonorthogonal sets was dimensional (Imai \& Garner, 1968) and dimensional preferences were found for orthogonal sets (Imai \& Garner, 1965; Handel, 1967). For Munsell colors, classification was highly correlated to similarity and preferences were not found (Handel, 1967).

The purpose of the present experiment was to investigate further the classification of analyzable and unanalyzable stimuli. For some sets, dimensional and similarity classification were placed in conflict to determine which classification was chosen; for other sets, dimensional classification by similarity was placed in conflict with dimensional classification by preference.

A priori, there is no reason for classification of analyzable and unanalyzable stimuli to differ. Analyzable stimuli, fitting the city-block metric, could be classified by the dimensional or similarity structure. Unanalyzable stimuli, fitting the euclidean metric, could also be classified by either structure. However, if the analyzable-unanalyzable distinction reflects the basic mode of perceiving multidimensional stimuli-either as an analyzable set of component dimensions or as an unanalyzable unitary complex dimension-then classification should differ. For analyzable stimuli, dimensional classification should predominate and dimensional preferences should occur, since the difference between stimuli is based on the dimensional structure. For unanalyzable stimuli, similarity classification should predominate and preferences should not occur, since the only meaningful difference between stimuli is dissimilarity.

\section{Stimulus Sets and Classifications:} Some Definitions

Orthogonal sets. Orthogonal sets contain four stimuli, the factorial combination of two levels of two dimensions. These sets can be classified into two groups of two stimuli on the basis of either dimension [e.g., (blue circle, blue square)/(green circle, green square) or (blue circle, green circle)/(blue square, green square)]. Furthermore, some sets of three stimuli can also be classified into two groups using either dimension (e.g., blue square, blue triangle, green square).

For all such sets, two-group dimensional classification is always the optimal similarity classification. Therefore, orthogonal sets cannot distinguish between dimensional and similarity classification. However, orthogonal sets can distinguish between dimensional classification based on similarity or based on preference. To ascertain the existence of dimensional preferences, at least two orthogonal sets must be used. In Set 1 , the levels of Dimension $x$ would be more dissimilar than the levels of Dimension y, but in Set 2, the dissimilarities would be reversed. If $S$ classifies Set 1 using Dimension $x$ and Set 2 using Dimension $y$, then $S$ is classifying by similarity. If $\mathrm{S}$ uses the same dimension to classify both sets, then $\mathbf{S}$ is demonstrating a dimensional preference.

Nonorthogonal sets. All nonorthogonal sets of three or four stimuli which fit into a 2 by 4 matrix can be classified dimensionally into two groups using only one dimension. For some sets, dimensional classification results in a balanced $2 / 2$ split [e.g., (blue circle, blue square)/(green triangle, green circle)] For other sets, dimensional classification results in an unbalanced 3/1 split [e.g., (blue circle, blue square, blue triangle)/(green circle)] For nonorthogonal sets, the optimal similarity classification may be nondimensional. Consider a set of three stimuli in which $A$ and $B$ differ on one dimension, $A$ and $C$ differ on two dimensions, but $A$ is more similar to $\mathbf{C}$ than $\mathbf{B}$. Dimensional classification would entail grouping $A$ and $B$ similarity classification would entail grouping $A$ and $C$.

There are several types of nondimensional classifications. In the first, termed bidimensional classification, the stimuli in each group differ on one dimension, but it is a different dimension for each group [e.g., (blue circle, green circle)/(blue square, blue triangle)]. Thus, bidimensional classification is similar to dimensional classification in that stimuli in each group differ on only one dimension; Ss are obviously using dimensions to form groups. But for bidimensional classification, the levels of one dimension do not define the groups. In the other nondimensional classifications, the stimuli in a group differ on both dimensions. These nondimensional classifications may be balanced [e.g., (blue circle, blue square)/(green circle, blue triangle)] or unbalanced [e.g., (blue circle, blue square, green circle)/(blue triangle)].

\section{Method}

Subjects. All 76 Ss were Kansas State University undergraduates.

Stimulus sets. Each dimension pair may be represented by a 4 by 4 matrix, with the rows and columns representing the four levels of each dimension (Fig. 1). Every set of three or four stimuli, therefore, may be portrayed by dots in the appropriate cells symbolizing the set stimuli. To 
limit the number of sets, only sets which use stimuli from one-half of the matrix (a 2 by 4 submatrix) were extensively investigated. That is, sets which fit in all six possible 2 by 4 submatrices were used, but sets which fit in a 3 by 3 submatrix (e.g., $/ 3 / 4$, $/ 3 / 8$, and $/ 5 / 8$ ) were not.

Furthermore, many sets are equivalent in that the structure among the stimuli is identical, although the actual stimuli differ. For the scalable dimension pairs (size by lightness, value by chroma), sets are equivalent if the dimensional relations among the stimuli are identical (i.e., dimensional structure) and the types of similarity differences among stimuli are identical (i.e., the similarity structure). This equivalence may be visualized: Let the three or four dots symbolizing one set be a visual pattern. This pattern can be moved as a unit in the matrix. Equivalent sets can be produced by translating, reflecting, and/or rotating by 90 or 270 deg the "dot pattern" inthe 4 by 4 matrix. In addition, for the shape by color stimuli, sets are equivalent if they can be produced by interchanging the nominal levels of each dimension.

On the basis of the logical structure, all possible stimulus sets were partitioned into a small number of unique sets. Each possible set was equivalent to only one of the unique sets. Logically equivalent sets, however, may not be classified identically. To demonstrate that an interaction between equivalents of a unique set and classification does not exist, all equivalents should be classified in the same manner (i.e., by the dimensional structure or by the similarity structure). This suggests an experimental procedure. For each unique set, present at least two equivalent sets to determine if each is classified identically. If not, present other equivalents of the same unique set to obtain reliable estimates of the percentages of differing classifications.

There were 11 sets of three stimuli. One set contained two identical stimuli and one stimulus differing by one level on one dimension. Ten sets were the unique sets in a 2 by 4 matrix, 2 sets containing stimuli differing on one dimension and 8 sets containing stimuli differing on two dimensions.

There were 24 sets of four stimuli. Nineteen sets were the unique sets, 1 set containing stimuli differing on one dimension and 18 sets containing stimuli differing on two dimensions. The remaining 5 sets were chosen to place dimensional and similarity classification in conflict.

Procedure. The $\mathbf{E}$ faced $\mathbf{S}$ across a table and placed the stimuli in front of S. For sets of three stimuli, S placed the stimuli into two groups in any way he wished. For sets of four stimuli, S was free to place the stimuli into two or three groups in any way he wished. Following this, $S$ was required to classify into two and three groups. The classification into the same number of groups as used for free classification was not repeated, since it was assumed that $\mathbf{S}$ would have repeated his free classification.

Experimental design. There were three experimental segments. In the first, two equivalents for every unique set were presented. For nonorthogonal sets, one equivalent could be dimensionally classified using the row dimension and one equivalent could be dimensionally classified using the column dimension. For orthogonal sets, the two equivalents reversed the similarity between levels on each dimension. Each equivalent was classified by $12 \mathrm{Ss}$, so that $24 \mathrm{Ss}$ classified each unique set. A total of 48 Ss was used.

The second and third segments were designed to explicate the results from the first segment. The second segment further sampled equivalents of those nonorthogonal sets in which the preferred classification was ambiguous. Across the four dimension pairs, there was a total of 120 sets. These sets were split in half, and six Ss classified each group of 60 sets. Analyses using both segments indicated that equivalents of each unique set were classified identically, so that the classifications were combined across equivalents. The third segment further sampled equivalents of orthogonal sets. Four equivalents for Sets $4 \mathrm{~N}$ and 40 were constructed (i.e., orthogonal sets in which row and column similarities were not equal) for each dimension pair. Sixteen Ss classified all equivalents.

For all segments, each unique set was always represented by an even number of equivalents, thereby balancing dimensional and similarity structure. Furthermore, for all segments, sets from different dimension pairs were alternated and the order of presentation was counterbalanced across Ss.

\section{Results}

Classification may be affected by the dimensional structure, by the similarity structure, and by dimensional preference. To differentiate among these factors, the stimulus sets are shown in two different figures.

Classification of nonorthogonal sets into two groups is shown separately for each dimension pair in Fig. $3 .^{2}$ If two-group dimensional classification was possible, the percentage of dimensional classifications as well as the percentage of classifications for the two optimal similarity classifications is shown. The similarity criteria was the smallest ratio of average within-group similarity divided by average between-group similarity (using thejudgments from Experiment 1.$)^{3}$ If dimensional classification was impossible, the percentages for the three optimal similarity classifications are shown. Bidimensional classifications are indicated by asterisks. Not all sets are shown in Fig. 3. These sets were chosen to be representative and to distinguish most clearly between dimensional and similarity classification.

Dimensional classification of orthogonal sets is shown in Fig. 4. For each set, the percentage of classifications into two groups using each dimension is shown for the two equivalents which reverse the similarity structure. If the dimensions are equally similar (Sets $3 F, 4 M$ ), similarity cannot provide a differential basis for classification.

One-dimensional sets. If sets contained two identical stimuli (Set 3A), the identical stimuli were invariably paired $(90 \%)$. If sets contained three different stimuli (Sets 3B-C), 80\% of the classifications paired the two most similar stimuli for the scalable dimensions. The shape and color dimensions were nominal, all pairs were equally similar, and no distinction was possible.

For sets of four stimuli, two-group classification resulted in a $2 / 2$ split. For the nominal shape and color dimensions, each of the three possible $2 / 2$ splits was equivalent, and these accounted for $70 \%$ of the classifications. For scalable dimensions, pairs of stimuli on adjacent levels were grouped (60\%). An unbalanced 3/1 classification was not preferred.

It must be concluded that the classification of one-dimensional sets was the same for all dimensions, with similarity and numerical balance influencing classification most, and the degree of discriminability not at all. ${ }^{4}$ Therefore, if the classification of two-dimensional sets differs, it cannot be attributed to classification of each dimension separately. Analyzability refers to a relation between dimensions and is not a property of a single dimension.

Two-dimensional sets: Dimensional us similarity classification. Two-dimensional sets can contrast dimensional and similarity classification for the size by lightness andthe value by chroma sets. Unfortunately, it is impossible to place dimensional and similarity classification in conflict for the shape 


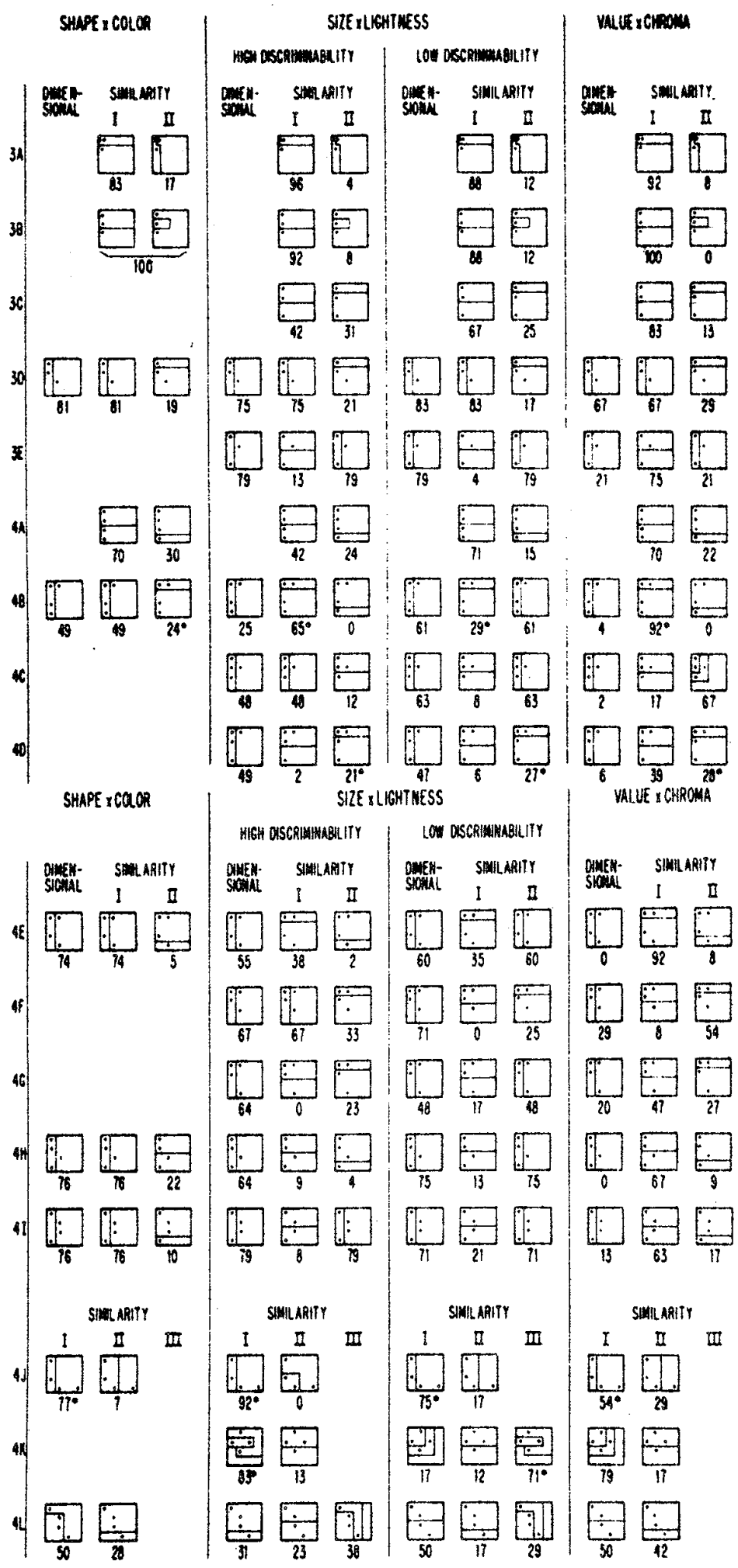

Fig. 3. The classification of one- and two-dimensional sets. For each unique set, the percentage of dimensional classifications and the percentage of similarity classifications are shown. Dots represent the stimuli in each set, and the inner lines indicate the classification. If dimensional classification of sets of four stimuli was impossible, only the similarity classifications are shown. Bidimensional classifications are indicated by asterisks. Many unique ets are equivalent for the nominal shape by color stimuli. Therefore, for the shape by color sets, the percentage of classifications is shown for only one of the equivalent unique sets. by color sets, because, for these stimuli, one-dimensional similarities were always greater than $t w o-d i m e n s i o n a l$ similarities. Thus, dimensional classification (grouping stimuli differing on one dimension) was always the optimal similarity classification. Nevertheless, the shape by color sets can serve as a baseline for the classification of other two-dimensional stimuli.

Two-group clansification of the shape by color sets was dimensional, dimensional classification being also the optimal similarity classification. For sets of three atimuli, $81 \%$ of the classifications were dimensional. For sets of four stimuli, $72 \%$ of the clamifications were dimensional. If dimensional classification was imposaible (Sets 4.J-K), bidimensional claseification was chosen (77\%).

For the size by lightneas sets, it was ponsible to place dimensional and similarity claseification in conflict. For both discriminabilities, two-group classification was dimensional; the similarity structure did not materially affect claseification. For sets of three stimuli, $80 \%$ of the classifications were dimensional, while for sets of four stimuli, $61 \%$ of the classifications were dimensional. For only one set (Set 4B, high discriminability) was the dimensional classification, not the preferred one.

The preferred alternative to two-group dimensional claseification was bidimensional classification. For Sets $4 B-D, 35 \%$ of the clasdifications were bidimentional. If bidimensional clasaification was impossible (Sets $4 E-G)$, the alternative paired two stimuli differing on one dimension and two stimuli differing on two dimensions (20\%). A 3/1 split was the least preferred classification, even if it was the optimal similarity classification (5\%). For both discriminabilities, if dimensional claspification was imposuible (Sets 4J-K), bidimensional classification was chosen $(80 \%)$, even if it was not the optimal similarity classification (Set 4K, low discriminability).

On the other hand, for the value by chroma sets, classification was based on similarity and numerical balance. Dimensional clastification was chosen only if it was the optimal similarity classification. For sets of three stimuli, $75 \%$ of the clasuifications were the optimal similarity classification. For sets of four stimuli, if the optimal similarity claseification was balanced (either dimensional, bidimensional, or nondimensional), $73 \%$ of the classifications were this type. If the optimal similarity classification was unbalanced, only $30 \%$ of the classifications were this type. However, as an unbalanced classification became more optimal by 
the similarity criteria, this classification became preferred. This can be seen for Sets $4 F$ and $4 G$, where the percentage increased from $8 \%$ to $47 \%$.

Two-dimensional sets: Dimensional preferences. Each of the 16 Ss classified four equivalents of Sets $4 \mathrm{~N}$ and 40 , so that it is possible to determine if dimensional preferences exist. Dimensional preference was defined as clansifying six or more of the eight equivalents by the same dimension.

Preferences existed for the shape by color sets: three Ss preferred the shape dimension (92\% of their classifications used shape), while three Ss preferred the color dimension $179 \%$ of their classifications used color). Overall, there was a slight preference for color classification $(49 \%$ color to $39 \%$ shape), although in Experiment 1 shape differences were judged twice as dissimilar as color differences.

Preferences also existed for the size by lightness sets. Six Ss preferred the lightness dimension (88\% lightness classification) and three Ss preferred the size dimension $(81 \%$ size classification). If similarity on each dimension was equal (Sets 3F, 4M), $72 \%$ of two-group classifications used the lightness dimension. Even if size differences were very much greater than lightness differences (Sets $3 \mathrm{H}$, 40 ), still $56 \%$ of the classifications used the lightness dimension. Thus, Ss preferred to classify on the basis of the lightness dimension, even when this placed very dissimilar stimuli in the same group. The strength of this preference diminished if size classification was a very optimal similarity classification.

Preferences were not found for the value by chroma sets. Classification was done simply on the basis of judged similarity; only three Ss showed preferences for one dimension, while eight Ss classified all equivalents by similarity.

\section{Summary}

The classification of the shape by color sets was dimensional, and preferences for dimensions existed. For these stimuli, two results suggested that the dimensional structure was more important than the similarity structure. First, two-group dimensional classification was twice as frequent as bidimensional classification ( $58 \%$ to $24 \%$ ), although both are nearly identical by the similarity criteria. Second, three-group dimensional classification [e.g., (blue circle, blue square)/(green circle)/(yellow circle)] was three times as frequent $(69 \%$ to $20 \%$ for Sets $4 \mathrm{~B}-\mathrm{D})$ as nondimensional classifications pairing stimuli differing

\begin{tabular}{|c|c|c|c|c|c|c|c|c|c|c|}
\hline \multirow{5}{*}{$\begin{array}{l}\text { Unique } \\
\text { Set }\end{array}$} & \multirow{4}{*}{\multicolumn{2}{|c|}{ Clossification }} & \multirow{3}{*}{\multicolumn{2}{|c|}{$\begin{array}{l}\text { SHAPE } \\
\text { COLOR }\end{array}$}} & \multirow{2}{*}{\multicolumn{4}{|c|}{$\frac{\text { SIZE } \times \text { LIGHTNESS }}{\text { DISCRIMINABILITY }}$}} & \multirow{3}{*}{\multicolumn{2}{|c|}{$\begin{array}{l}\text { VALUE } \\
\text { CHROMA }\end{array}$}} \\
\hline & & & & & & & & & & \\
\hline & & & & & \multicolumn{2}{|c|}{ HIGH } & \multicolumn{2}{|c|}{ Low } & & \\
\hline & & & \multicolumn{2}{|c|}{ Shope Color } & \multicolumn{2}{|c|}{ Size Lightness } & \multicolumn{2}{|c|}{ Size Lightmess } & \multicolumn{2}{|c|}{ Volue Chrome } \\
\hline & $I$ & II & $I$ & II & I & II & 1 & I & $I$ & $\mathbf{n}$ \\
\hline $3 F$ & E & & $\int$ & & 33 & 63 & 17 & 83 & 58 & 33 \\
\hline \multirow[t]{2}{*}{36} & E & $E$ & & & 17 & 83 & 17 & 78 & 33 & 50 \\
\hline & 用 & $F$ & 39 & 50 & 33 & 61 & 33 & 56 & 58 & 42 \\
\hline \multirow[t]{2}{*}{$3 H$} & & $B$ & & & 50 & 50 & 44 & 50 & 8 & 83 \\
\hline & ET & & l & & 25 & 67 & 11 & 78 & 92 & 8 \\
\hline MEAN & & & 39 & 50 & 32 & 65 & 23 & 71 & 51 & 42 \\
\hline $4 M$ & 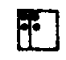 & H & $\int$ & & 17 & 75 & 21 & 67 & 63 & 25 \\
\hline \multirow[t]{2}{*}{$4 N$} & (2.) & 昆 & & & 27 & 61 & 25 & 57 & 9 & 75 \\
\hline & 80 & $\theta$ & 39 & 47 & 32 & 57 & 27 & 50 & 69 & 12 \\
\hline \multirow[t]{2}{*}{40} & [a] & $\theta$ & & & 27 & 57 & 39 & 41 & 19 & 72 \\
\hline & T3 & 9 & & & 36 & 39 & 34 & 57 & 81 & 9 \\
\hline MEAN & & & 39 & 47 & 26 & 61 & 28 & 57 & 57 & 36 \\
\hline
\end{tabular}

Fig. 4. Dimensional classification of sets of three or four stimuli which can be classified using either the row or column dimension. In the first column, the unique set and the classification using the row or column are indicated. If the similarity between levels of each dimension is not equal, the classification of the two equivalents which reverse the dimensional structure are shown. In the remaining columns, the percentage of times each dimension was chosen for classification is shown for each dimension pair. For the nominal shape by color dimensions, Sets $3 F-3 H$ and Sets $4 M-4 O$ are logically equivalent.

on one dimension [e.g., (blue circle, green circle $) /($ yellow circle $) /($ blue square)], although these classifications were equal by the similarity criteria.

The classification of the size by lightness sets was dimensional, and preferences were also found. Similarity affected classification only within the dimensional structure. First, dimensional classifications which were also the optimal similarity classifications were chosen slightly more frequently than dimensional classifications which were not (discussed later in Table 2). Second, although an optimal similarity bidimensional classification could be preferred to a dimensional classification ( Set $4 \mathrm{~B}$, high discriminability), other nondimensional classifications which grouped stimuli differing on two dimensions, regardless of similarity, were rarely chosen. Third, for orthogonal sets, similarity could only weaken dimensional preferences. However, discriminability did not affect classification.

In contrast, the classification of the value by chroma sets was based on similarity, with Ss exhibiting little in the way of dimensional preferences. However, Ss preferred balanced classifications to unbalanced classifications, sometimes thereby choosing a nonoptimal similarity classification (e.g., Sets 4C, 4F).

These results demonstrate two 
Table 2

Average Percentage of Clasaification for Each Caterory

\begin{tabular}{|c|c|c|c|c|c|}
\hline \multirow[b]{3}{*}{ Dimension-Pair } & \multirow{3}{*}{$\begin{array}{c}\text { Numerical } \\
\text { Balance }\end{array}$} & \multicolumn{4}{|c|}{ Classification } \\
\hline & & \multicolumn{2}{|c|}{ Dimensional } & \multicolumn{2}{|c|}{ Nondimensional } \\
\hline & & Nonsimilax & Similar & Nonsimilar & Similar \\
\hline Shape by Color & $\begin{array}{l}3-1 \\
2-2\end{array}$ & - & $\begin{array}{l}64 \\
75\end{array}$ & $\begin{array}{r}6 \\
25\end{array}$ & - \\
\hline Size by Lightness & $\begin{array}{l}3-1 \\
2-2\end{array}$ & $\begin{array}{l}55 \\
65\end{array}$ & $\begin{array}{l}63 \\
69\end{array}$ & $\begin{array}{r}3 \\
24\end{array}$ & $\begin{array}{r}6 \\
28\end{array}$ \\
\hline Value by Chroma & $\begin{array}{l}3-1 \\
2-2\end{array}$ & $\begin{array}{r}5 \\
\mathbf{2 0}\end{array}$ & $\begin{array}{l}25 \\
82\end{array}$ & $\begin{array}{r}7 \\
43\end{array}$ & $\begin{array}{l}27 \\
77\end{array}$ \\
\hline
\end{tabular}

general principles: First, Ss prefer numerically balanced classifications (see also Imai, 1966). Averaged across all sets, $72 \%$ of two-group classifications split the stimuli into two groups of two stimuli. Second, Ss try to classify sets of stimuli so that the stimuli in each group differ on only one dimension. Dimensional classifications are of this type, but so are bidimensional classifications. For analyzable stimuli (shape by color, size by lightness), dimensional was preferred to bidimensional classification. If Ss did not use dimensional or bidimensional classification, they classified so that the stimuli in one group differed on one dimension. For the unanalyzable stimuli used (value by chroma), differences between dimensional, bidimension al, and $2 / 2$ nondimensional classifications should not be found, and results did, indeed, show the effects only of similarity.

In summary, a ranking of classifjcations exists for analyzable stimuli: dimensional classification was preferred, bidimensional classification was next, $2 / 2$ nondimensional classification was third, and $3 / 1$ nondimensional classifiation was least preferred. On the other hand, for unanalyzable stimuli, balanced similarity classification was preferred to unbalanced similarity classification.

\section{DISCUSSION}

Perception of Dimensions

As expected, similarity judgments differentiated among dimension pairs on the basis of stimulus analyzability. For shape by color and size by lightness stimuli, Ss seemed to judge similarity by combining differences along each dimension. For value by chroma stimuli, Ss seemed to judge differences between stimuli directly.

The classification task produced the same pattem of results. Classification of shape by color and size by lightness stimuli was based on dimensional structure, with obvious dimensional preferences in evidence. Classification of the value by chroma was based on similarity, and no dimensional preferences appeared.
Discriminability did not affect either similarity judgments or classification of the size by lightness stimuli. For both high and low discriminability, these stimuli remained analyzable. In a speeded classification task, Garner and Felfoldy (1970) found that value by chroma stimuli were unanalyzable for both high and low discriminability. Thus, analyzability seems independent of discriminability, and it appears impossible to vary stimulus analyzability by varying stimulus discriminability.

To conclude, shape by color and size by lightness stimuli are perceived as two-dimensional stimuli; Ss often utilize each dimension separately in making similarity judgments and classifications. Value by chroma stimuli are not perceived as two-dimensional stimuli; $S$ s seem unaware of their dimensional properties and make similarity and classificatory judgments directly on the basis of perceived difference. This is not to say that Munsell colors cannot be perceived dimensionally, for, after all, the Munsell system was constructed by observers who judged differences in hue, value, and chroma separately (Newhall, 1940). Practice or explicit instructions can bring about dimensional perception.

\section{Relation Among Dimensionality,}

Numerical Balance, and Similarity

Our premise is that under optimal conditions, Ss try to group four stimuli so that the classification is dimensional, is numerically balanced, and so that similar stimuli are placed in the same group. The simplest model proposes that these factors-dimensionality, balance, and similarity-are additive in determining the percent choice of each classification. In other words, each factor may be positive or negative (i.e., dimensional or nondimensional, balanced or unbalanced, similar or nonsimilar), and each positive factor adds to the percent choice of that classification.

To determine the role of each factor, two-group classifications were split into eight categories. Four categories were dimensional classifications, four categories were nondimensional classifications. The four categories for both dimensional and nondimensional classification were formed on the basis of whether two-group classification resulted in a balanced or an unbalanced classification and whether the classification was the optimal similarity or nonoptimal similarity classification. For nondimensional classifications, the nonsimilar classification was the second best similarity classification.

For each category and dimension pair, the average percentage of classification across all two-dimensional sets of four stimuli is shown in Table 2. For the shape by color sets, dimensional classification must be the optimal similarity classification. The comparisons thvolve only numerical balance. The difference between balanced and unbalanced classifications was $9 \%$ for dimensional classification and $19 \%$ for nondimensional classification.

For the size by lightness sets, the factors of dimensionality, balance, and similarity were additive, although not equally important. Dimensionality was the most important factor. The difference between dimensional and nondimensional classifications was $49 \%(64 \%$ to $15 \%)$, the difference between balanced and unbalanced classifications was $14 \%$ (46\% to $32 \%)$, and the difference between similar and nonsimilar classifications was only $4 \%$ (42\% to $38 \%$ ). There was a slight interaction between dimensionality and balance; the difference between balanced and unbalanced classifications was $8 \%$ for dimensional classification, but $21 \%$ for nondimensional classifications. However, the difference between similar and nonsimilar classifications was identical and small, regardless of whether the classifications were dimensional or balanced.

For the value by chroma sets, balance and similarity interacted, but dimensionality did not affect classification. For unbalanced classifications, the difference between similar and nonsimilar classifications was $20 \%$ (26\% to $6 \%$ ), but for balanced classifications, the difference between similar and nonsimilar classifications was $48 \%$ ( $80 \%$ to $32 \%$ ). Dimensionality did not affect classification; in fact, nondimensional classifications were slightly more frequent ( $39 \%$ to $33 \%$ ).

To summarize, for the shape by color and the size by lightness sets, dimensionality was the most important factor, accounting for $50 \%$ of the classifications. Balance was an additive factor, accounting for about 
$15 \%$ of the classifications. In addition, for the size by lightness sets, similarity was also additive, accounting for $4 \%$ of the classifications. In contrast, for the value by chroma sets, similarity and balance interacted in determining classification; dimensionality did not affect classification.

\section{The Genesis of Structure}

To determine the principle used for two-group classification, a set of at least three stimuli is necessary. Since any set of four or more stimuli can be broken into subsets of three stimuli, the classification of sets of three stimuli should serve as a basis for understanding the classification of larger sets. In the present experiment, it was possible to compare the classification of sets of three stimuli with the classification of the identical three stimuli embedded in a set of four stimuli. If the classifications are identical, then a set of three stimuli provides a structure for classification within which additional stimuli are incorporated. If the classifications are not identical, each additional stimulus can bring about a new structure for classification.

The comparison between the classification of a three-stimulus subset and a set of three stimuli was all or none. On the basis of the classification of the set of four stimuli, the classification of each subset of three stimuli was identified. Once this was done, the percentage of times the classification of the subset was the same as the identical set of three stimuli was calculated.

Two-dimensional sets may contain one-dimensional subsets. The classification of one-dimensional sets was done on the basis of similarity and balance. For the two-dimensional shape by color and size by lightness sets, dimensional classification predominated; three stimuli differing on one dimension were grouped together regardless of the similarity among these stimuli. Therefore, the classification of stimuli varying on one dimension differed for one and two-dimensional sets. For two-dimensional value by chroma sets, similarity classifications predominated; three dissimilar stimuli, although differing on only one dimension, were placed in different groups. Therefore, the classification of stimuli varying on one dimension was identical.

Two-dimensional sets may contain two-dimensional subsets. For shape by color sets, classification $w$ as dimensional. Thus, there was $100 \%$ agreement between the classification of two-dimensional subsets of three stimuli and two-dimensional sets of three stimuli. For the size by lightness sets, $98 \%$ of the corresponding subsets and sets were classified identically. For the value by chroma sets, there was only 72\% agreement. Although not changing pairwise similarities, introducing one additional stimuli altered the overall pattern of similarities and thereby changed classification. For example, consider Set 4I. This set contains two equivalents of Set $3 D$ and Set $3 E$. When presented alone, Set 3D was classified by similarity; when embedded in Set 4I, however, Set 3D was classified by placing dissimilar stimuli in the same group. The preferred classification of Set $4 I$ optimized similarity among the four stimuli, although the optimal similarity classification did not occur for each subset of three stimuli.

To conclude, the basis of classification differs for analyzable and unanalyzable stimuli. For analyzable stimuli, the introduction of a stimulus which redefines the dimensional structure alters classification. Stimuli which specify each level of all dimensions provide a basis for classification. A stimulus which defines a new level may alter classification, but a stimulus which fits within a preexisting dimensional structure will not alter classification. For unanalyzable stimuli, the introduction of a stimulus which redefines the similarity structure changes classification. Stimuli which define distances provide a basis for classification. A stimulus which transforms the pattern of similarities among stimuli will alter classification. $A$ set of stimuli formed from unanalyzable stimuli cannot be conceptualized solely in terms of the individual stimuli.

\section{REFERENCES}

GARNER, $W$. R. \& FELFOLDY, G. L Integrality of stimulus dimensions in various types of information processing. Cognitive Psychology, 1970, 1, 225-241.

HANDEL, $S$. Classification and similarity of multidimensional stimuli. Perceptual \& Motor Skills, 1967, 24, 1191-1203.

HYMAN, R., \& WELL, A. Judgments of similarity and spatial models. Perception \& Psychophysics, 1967, 2, 233-248.

HYMAN, R., \& WELL, A. Perceptual separability and spatial models. Perception \& Psychophysics, 1968, 3, 161-165.

IMAI, S. Classification of sets of stimuli with different stimulus characteristics and numerical properties. Perception \& Psy chophysics, 1966, 1, 48-54.

I MAI, S..\& GARNER, W. R. Discriminability and preference for attributes in free and constrained classification. Journal of Experimental Psychology, 1965, 69, 596-608.

IMAI, S., \& GARNER, W. R. Structure in perceptual classification. Psychonomic Monograph Supplements, 1968, 2(9, Whole No. 25).

MICKO, H. C., \& FISCHER, W. The metric of multidimensional psychological spaces as a function of the differential attention to subjective attributes. Journal of Mathematical Psychology, 1970, 7 . 118-143.

NEWHALL, S. M. Preliminary report of the O.S.A. subcommittee on the spacing of the Munsell colors. Journal of the Optical Society of America, 1940, 30, 617-645. TORGERSON, W. S Theory and methods of scaling. New York: Wiley, 1958.

\section{NOTES}

1. We are grateful to Mr. Dennis Patterson, Miss Anne Kaiser, and Mr. Herb Bell for collecting and tabulating the data.

2. Only two-group classification is extensively discussed, since identical conclusions regarding classification can be drawn from three-group classification.

3. The ratio of within-group similarity to between-group similarity was calculated as follows: For sets of three stimuli, two-group classification results in one similarity within-groups and two similarities between-groups. Clearly, the optimal similarity classification is one in which the two most similar stimuli are paired. For sets of four stimuli, there are six similarities among the four stimuli. For a 2/2 classification, two similarities are within-groups and four similarities are between-groups: for a $3 / 1$ classification. three similarities are within-groups and three similarities are between-groups (i.e., a group of one stimulus is not considered to have within-group similarity). For three-group classification (e.g., 2/1/1), one similarity is within-groups and five similarities are between groups. For all classifications, the average similarity within-groups and average similarity between-groups were found and the ratio calculated.

4. A manuscript in preparation will further detail the classification of one-dimensional sets.

(Accepted for publication April 5, 1972.) 